\title{
Tostación de un concentrado refractario de oro y plata ${ }^{(\cdot)}$
}

\author{
J. H. Coronado*, M. A. Encinas*, J. C. Leyva*, J. L. Valenzuela*, A. Valenzuela* y G.T. Munive* \\ Resumen \\ En el procesamiento de minerales de metales preciosos con altos contenidos de pirita, se obtienen concentrados \\ refractarios difíciles de procesar. En este estudio se lixivió un concentrado refractario de oro y plata con cianuro de \\ sodio, obteniéndose extracciones de 34 \% para oro y $40 \%$ para plata. Se utilizó el método de tostación para oxidar \\ el concentrado, haciéndolo más susceptible a la cianuración, y extraer más eficientemente el oro y la plata. Las \\ variables analizadas fueron: temperatura de tostación y tiempos de tostación y cianuración. Además, la calcina caliente \\ se agregó a la solución lixiviante a temperatura ambiente para analizar el efecto en el tamaño de partícula y recuperación. \\ Los mejores resultados, aunque no del todo satisfactorios ( $50 \%$ oro y $61 \%$ plata), se obtuvieron mediante tostación \\ del concentrado durante $4 \mathrm{~h}$ a temperaturas de $600^{\circ} \mathrm{C}$, seguido de una cianuración de $20 \mathrm{~h}$. El consumo de cal para \\ elevar el pH a 11,3, se incrementó notablemente hasta $25 \mathrm{~kg} / \mathrm{m}^{3}$.
}

Palabras clave Tostación; Minerales refractarios; Cianuración.

\section{Roasting of refractory gold and silver concentrate}

\begin{abstract}
In processing of precious metal ores with high pyrite content, refractory concentrates are obtained, which are difficult to process. A refractory gold and silver concentrate was leached with sodium cyanide. Results show low extraction percentages, being $34 \%$ of gold and $40 \%$ of silver. A roasting method to oxidize the concentrate was used, making it more susceptible to cyanidation, hence a more efficient way to extract precious metals. The variables include roasting temperature and roasting and cyanidation time. In addition, the hot calcine was added to the leaching solution at room temperature to analyze the effect on particle size and recovery. The best results, although not entirely satisfactory ( $50 \%$ of gold and $61 \%$ of silver) were obtained by roasting the concentrate for $4 \mathrm{~h}$ at $600^{\circ} \mathrm{C}$, followed by cyanidation for $20 \mathrm{~h}$. The lime consumption to raise the $\mathrm{pH}$ to about 11.3 was increased markedly to $25 \mathrm{~kg} / \mathrm{m}^{3}$.
\end{abstract}

Keywords Roasting; Refractory minerals; Cyanidation.

\section{INTRODUCCIÓN}

En México existen muchas menas de baja ley, así como otras en las que el oro y la plata se encuentran ocluidos o asociados a minerales de hierro, arsénico, manganeso y silicio, los cuales se clasifican como depósitos refractarios auríferos y argentíferos. La mayoría de las veces, la cianuración de estos minerales requiere largos periodos de lixiviación y, desafortunadamente, bajas recuperaciones de oro ${ }^{[1-5]}$. La tostación de minerales y concentrados refractarios de oro, antes de la lixiviación con cianuro, es un método alternativo que se ha empleado durante décadas. El principal objetivo de la tostación es liberar el oro íntimamente asociado a los minerales sulfurosos y telurosos, para aumentar la extracción del oro durante la lixiviación con cianuro. Esto se realiza oxidando los sulfuros a óxidos o sulfatos, o reduciendo los silicatos y óxidos a un estado metálico crudo, con el propósito de cambiar los compuestos metálicos insolubles en otros que sean solubles en el agente lixiviante; así como para volatilizar ciertas impurezas solubles que pudieran contaminar la solución cargada. También es útil para volver porosos los compuestos metálicos, y hacerlos de esta forma más fácilmente accesibles al disolvente de la lixiviación ${ }^{[6]}$.

Como la tostación es una operación relativamente costosa, solo puede aplicarse a materiales de alta ley que puedan justificar los altos costos de este tratamiento. Sin embargo, puede obtenerse el efecto de

(•) Trabajo recibido el día 13 de enero de 2011 y aceptado en su forma final el día 20 de febrero de 2012.

* Departamento de Ingeniería Química y Metalurgia, Universidad de Sonora. Rosales y Blvd. Luis Encinas S/N, Hermosillo, Sonora, 83000, México. E-mail:(jhcoronado@iq.uson.mx. 
una tostación oxidante o sulfatizante de minerales sulfurosos de baja ley, mediante su intemperización natural experimentada durante un periodo prolongado de tiempo.

La mineralización de los materiales refractarios de oro no se ha establecido detalladamente para todos los depósitos; no obstante, en muchos depósitos, la resistencia a la cianuración directa se atribuye al oro extremadamente fino encapsulado en pirita y ciertos minerales de la ganga, como cuarzo. La presencia de materia carbonosa, arcillas, cianicidas, películas pasivantes, teluros y otros muchos factores pueden también propiciar la refractariedad de una mena ${ }^{[7]}$. En algunos depósitos se ha observado que el oro incrustado en pirita se presenta en una forma no metálica asociada a zonas ricas de arsenopiritas. Una explicación es que los átomos de oro han substituido al arsénico o a los posibles átomos de hierro, dando lugar a una adecuada substitución intersticial. Metalúrgicamente esto podría ser significativo, y puede explicar el porqué en una oxidación se proyecta solamente la oxidación parcial de los sulfuros ${ }^{[8]}$.

La química básica de una oxidación por tostación para minerales concentrados piritosos y arsenopiritosos de oro, es relativamente sencilla; los sulfuros metálicos son convertidos a óxidos metálicos y dióxido de azufre $\left(\mathrm{SO}_{2}\right)$, este último liberándose como gas. Las reacciones se desarrollan generalmente entre 600 y $700^{\circ} \mathrm{C}$.

La tostación de la pirita $\left(\mathrm{FeS}_{2}\right)$ en una atmósfera altamente oxidante, produce la hematita $\left(\mathrm{Fe}_{2} \mathrm{O}_{3}\right)$ según lo mostrado en la reacción (1):

$$
4 \mathrm{FeS}_{2}+11 \mathrm{O}_{2} \rightarrow 2 \mathrm{Fe}_{2} \mathrm{O}_{3}+8 \mathrm{SO}_{2}
$$

Cuando la atmósfera de la tostación es menos oxidante, la magnetita $\left(\mathrm{Fe}_{3} \mathrm{O}_{4}\right)$, será producida según lo mostrado en la reacción $(2)$ :

$$
3 \mathrm{FeS}_{2}+8 \mathrm{O}_{2} \rightarrow \mathrm{Fe}_{3} \mathrm{O}_{4}+6 \mathrm{SO}_{2}
$$

Para la extracción más favorable del oro, un producto que contiene aproximadamente 75 a $85 \%$ de hematita, y el resto magnetita, es generalmente el más deseable. Se puede formar durante la tostación un poco de sulfato de hierro $\left(\mathrm{FeSO}_{4}\right)$, el cual es generalmente indeseable, los ferrocianuros se forman fácilmente con $\mathrm{FeSO}_{4}{ }^{[9]}$.

La arsenopirita ( $\mathrm{FeAsS}$ ) es oxidada a dióxido de azufre $\left(\mathrm{SO}_{2}\right)$, hematita $\left(\mathrm{Fe}_{3} \mathrm{O}_{4}\right)$ y trióxido de arsénico $\left(\mathrm{As}_{2} \mathrm{O}_{3}\right)$.
La oxidación de la arsenopirita es algo más compleja que la de la pirita. La reacción (3) ocurrirá si la arsenopirita se tuesta en una atmósfera altamente oxidante.

$$
\mathrm{FeAsS}+3 \mathrm{O}_{2} \rightarrow \mathrm{FeAsO}_{4}+\mathrm{SO}_{2}
$$

Esta es una reacción indeseable puesto que se forma el arseniato férrico $\left(\mathrm{FeAsO}_{4}\right)$, que inhibirá la extracción del oro durante la lixiviación con cianuro. Las reacciones deseables son mostradas en las reacciones (4 y 5); la reacción (4) convierte el arseniato a trióxido de arsénico volátil $\left(\mathrm{As}_{2} \mathrm{O}_{3}\right)$, en una atmósfera menos oxidante a una temperatura relativamente más baja $\left(500{ }^{\circ} \mathrm{C}\right.$ y $80 \%$ de $\left.\mathrm{O}_{2}\right)$.

La reacción (5) se desarrolla a una temperatura y a una atmósfera altamente oxidante.

$$
\begin{gathered}
12 \mathrm{FeAs} \mathrm{S}+29 \mathrm{O}_{2} \rightarrow \\
6 \mathrm{As}_{2} \mathrm{O}_{3}+4 \mathrm{Fe}_{3} \mathrm{O}_{4}+12 \mathrm{SO}_{2} \\
4 \mathrm{Fe}_{3} \mathrm{O}_{4}+\mathrm{O}_{2} \rightarrow 6 \mathrm{Fe}_{2} \mathrm{O}_{3}
\end{gathered}
$$

Para lograr estas reacciones se requiere una tostación en 2 etapas. La etapa 1 debe ser a bajas temperaturas y poco aire para producir $\mathrm{As}_{2} \mathrm{O}_{3}$ y evitar la formación de $\mathrm{FeAsO}_{4}$. La etapa 2 debe ser a una temperatura alta y con exceso de aire para producir la hematita porosa ( $\mathrm{As}_{2} \mathrm{O}_{3}$ es durante la tostación).

La reacción (6) muestra que el carbón orgánico elemental $(\mathrm{C})$ se oxida a dióxido de carbono $\left(\mathrm{CO}_{2}\right)$.

$$
\mathrm{C}+\mathrm{O}_{2} \rightarrow \mathrm{CO}_{2}
$$

Esta reacción depende del tipo de carbón presente y de la temperatura de tostación. El carbón orgánico con una temperatura de ignición bastante baja se oxidará fácilmente a $\mathrm{CO}_{2}$, mientras que los carbonos de tipo grafíticos con altas temperaturas de ignición pueden no reaccionar durante la tostación. Los carbonatos de magnesio y calcio $\left(\mathrm{MgCO}_{3}, \mathrm{CaCO}_{3}\right)$, se calcinan parcialmente a óxidos; sin embargo, las temperaturas de tostación son demasiado bajas para la calcinación completa ${ }^{[6]}$.

La reacción (7) muestra la descomposición de los carbonatos de calcio y magnesio a óxidos de calcio y magnesio $(\mathrm{CaO} / \mathrm{MgO})$.

$$
\mathrm{CaCO}_{3} / \mathrm{MgCO}_{3} \rightarrow \mathrm{CaO} / \mathrm{MgO}+\mathrm{CO}_{2}
$$


TOSTACIÓN DE UN CONCENTRADO REFRACTARIO DE ORO Y PLATA ROASTING OF REFRACTORY GOLD AND SILVER CONCENTRATE

Esta reacción depende del tipo de carbonatos presentes y de la temperatura. El carbonato de magnesio se descompone a una temperatura mucho más baja que el carbonato de calcio $\left(350^{\circ} \mathrm{C}\right.$ frente a $825^{\circ} \mathrm{C}$, dependiendo de los minerales). La descomposición del dióxido de azufre descomponiéndose a sulfato o sulfito $\left(\mathrm{SO}_{3}{ }^{-}\right)$es a menudo una reacción deseable, porque reduce la cantidad de $\mathrm{SO}_{2}$ que se debe lavar en los gases de salida. La desventaja de la formación de sulfato de calcio $\left(\mathrm{CaSO}_{4}\right)$ es que pueden alterarse las características de la calcina e inhibir la disolución del oro.

La reacción (8) muestra la reacción entre $\mathrm{CaO}$ o $\mathrm{MgO}$, generado en la reacción (7), y el $\mathrm{SO}_{2}$ durante la tostación.

$$
\mathrm{CaO} / \mathrm{MgO}+\mathrm{SO}_{2}+1 / 2 \mathrm{O}_{2} \rightarrow \mathrm{CaSO}_{4} / \mathrm{MgSO}_{4}
$$

Esta reacción es generalmente deseable, sin embargo, con algunos minerales concentrados, la formación de sulfato de calcio inhibirá la extracción del oro durante la lixiviación con cianuro (el oro se encapsula en el sulfato de calcio). Esta reacción se refiere a la fijación del $\mathrm{SO}_{2}$. Una tostación ideal formará la hematita porosa; la temperatura excesiva de tostación (sobre tostación o sinterización del mineral), inhibirá la formación de la hematita y reducirá seriamente la extracción del oro. La tostación generalmente es perjudicial para la plata en las extracciones por lixiviación; cuanto más alta es la temperatura de tostación, más baja es la extracción de plata. Las variables de proceso más importantes que deben ser consideradas en la tostación son: cantidad de alimentación, temperatura, tiempo de tostación, adición de oxígeno, oxidación del azufre y del carbón orgánico elemental, fijación del dióxido de azufre, entre otras ${ }^{[5,10 \text { y } 11]}$.

\section{MÉTODO EXPERIMENTAL}

El análisis químico del concentrado mineral utilizado en este estudio se muestra en la tabla I. Las pruebas de tostación se llevaron a cabo utilizando un horno eléctrico marca Lindberg/Blue M., modelo 55345. Para hacer los análisis del tamaño de partícula, se utilizó un analizador de partículas marca Coulter 100 Q. Para evaluar los resultados obtenidos en la tostación del concentrado se utilizó una lixiviación agitada con cianuro de sodio $(\mathrm{NaCN})$, en un reactor de vidrio Pyrex de $100 \mathrm{ml}$ con acceso para la toma de muestra, y un sistema de agitación marca STIR-PAK de 25-2.300 rpm, equipado con flecha y propela. Los reactivos utilizados en la lixiviación fueron cianuro
Tabla I. Análisis químico del concentrado

Table I. Chemical analysis of concentrate

\begin{tabular}{cc}
\hline Elemento & Composición (\%) \\
\hline $\mathrm{Au}$ & $60^{(*)}$ \\
$\mathrm{Ag}$ & $10,200^{(*)}$ \\
$\mathrm{Fe}$ & 36,38 \\
$\mathrm{Cu}$ & 0,50 \\
$\mathrm{Zn}$ & 4,72 \\
$\mathrm{As}$ & 0,15 \\
$\mathrm{~S}$ & 33,34 \\
\hline $\mathrm{pH}$ & 6,05 \\
\hline
\end{tabular}

$\left.{ }^{*}\right) \mathrm{g} / \mathrm{t}$.

de sodio, hidróxido de calcio y nitrato de plata, todos ellos de grado reactivo y disueltos en agua destilada. Para el análisis de la solución lixiviada, se utilizó un espectrofotómetro de absorción atómica, marca Perkin Elmer modelo 3110 con lámpara de cátodo hueco a una longitud de onda de 242,8 nm para el oro; para la plata se aplicó una llama de oxígeno-acetileno y una longitud de $328,1 \mathrm{~nm}$.

En cada prueba experimental, primero se ajustó el horno tostador a temperatura de trabajo, posteriormente se pesaron $12 \mathrm{~g}$ de concentrado, los cuales se colocaron en un recipiente de porcelana; la muestra de concentrado se introdujo en el interior del horno tostador por un tiempo programado. Al finalizar la etapa de tostación, la muestra se retiró del horno, transfiriéndose a un reactor con el agente lixiviante, midiendo inmediatamente el valor de $\mathrm{pH}$. Seguidamente se adicionó cal hasta alcanzar un valor de $\mathrm{pH}$ entre 10,5 y 11,0 , para posteriormente agregar el cianuro de sodio, tomando este paso como el inicio de la etapa de lixiviación y manteniéndola durante el intervalo de tiempo determinado. Se tomaron muestras de solución a intervalos de tiempo predeterminados para analizar las concentraciones de oro y plata en la solución. La cantidad de $\mathrm{NaCN}$ en la solución, se obtuvo por titulación con nitrato de plata.

\section{RESULTADOS Y DISCUSIÓN}

En la figura 1 a) se presenta el análisis de tamaño de partícula del concentrado sin tostar. Podemos observar que la media del tamaño de partícula se encuentra alrededor de 23,56 micras, y la moda alcanza un valor de 23,81 micras. 


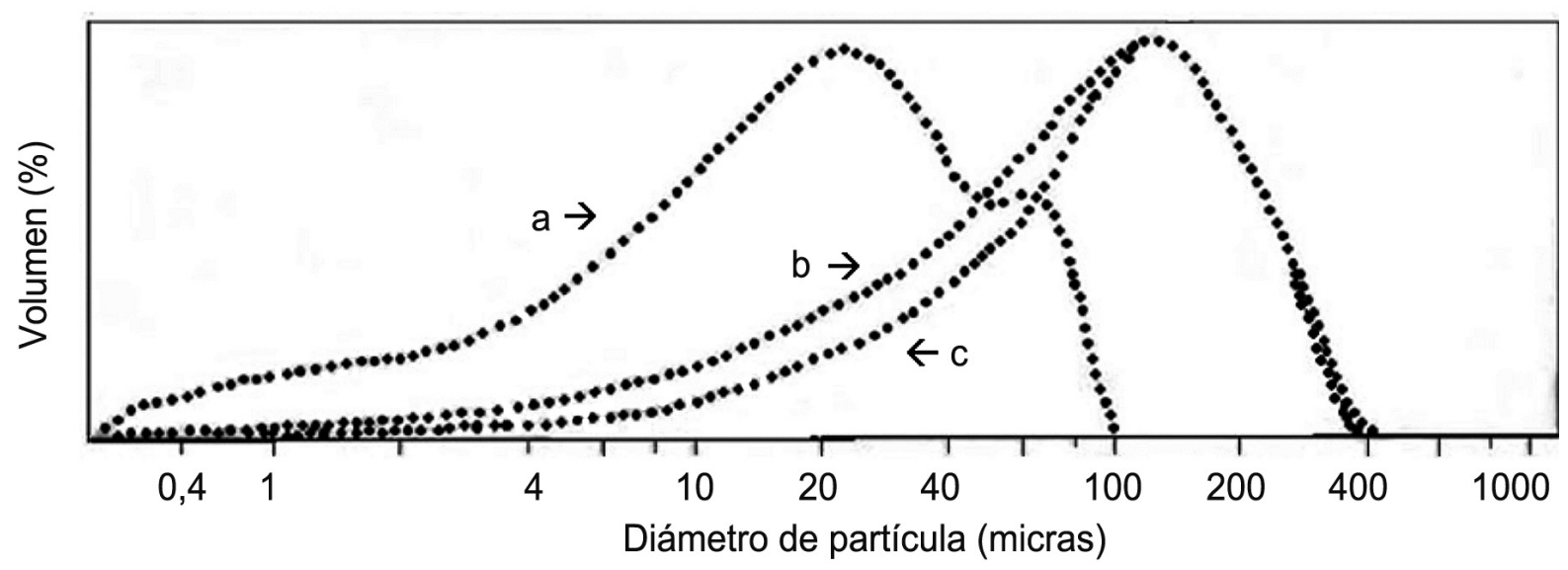

Figura 1. Resultados del analizador de partículas del concentrado: 1 a) sin tostar; 1 b) concentrado tostado, $\mathrm{t}=1 \mathrm{~h}, \mathrm{~T}=500^{\circ} \mathrm{C} ; 1 \mathrm{c}$ ) concentrado tostado, posteriormente la calcina caliente se agregó inmediatamente a la solución lixiviante, $\mathrm{t}=1 \mathrm{~h}, \mathrm{~T}=500^{\circ} \mathrm{C}$.

Figure 1. Particle size analyses of the concentrate: 1 a) unroasted concentrate; b) roasted concentrate, $t=1 \mathrm{~h}, T=500^{\circ} \mathrm{C} ; 1 \mathrm{c}$ ) roasted concentrate, after roasting the hot calcine was added immediately to the leaching solution, $t=1 \mathrm{~h}, \mathrm{~T}=500^{\circ} \mathrm{C}$.

Analizando de forma general, podemos concluir que el concentrado obtenido del proceso de flotación contiene partículas finas, con tamaños menores de 33 micras. En la figura 1 b) se presenta el análisis de tamaño de partícula obtenido del concentrado calcinado por un tiempo de $1 \mathrm{~h}$ a una temperatura de $500{ }^{\circ} \mathrm{C}$, con el objetivo de observar el cambio de tamaño de partículas al someterse al proceso de tostación. En esta figura se observa que existe un aumento considerable en el tamaño de la partícula después del proceso de tostación, ya que el valor medio en este caso resultó ser de 95,18 micras, con una moda de 127,6 micras. Por lo anterior se concluye que existe un aumento en el tamaño de las partículas del concentrado al estar expuesto a este tratamiento térmico, el cual posiblemente se deba a una expansión de las partículas, generada por la salida de elementos en forma de gases, los cuales tienen su punto de ebullición menor a $500^{\circ} \mathrm{C}$. En la figura $1 \mathrm{c}$ ), se presenta el análisis de tamaño de partícula del concentrado que fue sometido a un proceso de tostación a una temperatura de $500^{\circ} \mathrm{C}$ durante una hora, y posteriormente la calcina caliente se agregó inmediatamente a la solución lixiviante, que se encontraba a temperatura ambiente, para lograr con esto, un cambio rápido de temperatura en el sistema; con el objetivo de determinar si la partícula caliente, por efecto del cambio térmico sufre algún tipo de fragmentación en su tamaño, lo cual podría ayudar a la liberación de los valores. Sin embargo, comparando estos resultados con los de la figura $1 \mathrm{~b}$ ), observamos diferencias poco significativas en el tamaño de partícula; éstas se consideran no significativas para deter- minar que exista algún cambio apreciable en el tamaño de la partícula. La tabla II, presenta el análisis de los parámetros estadísticos antes mencionados.

Tabla II. Análisis estadístico del analizador de partículas

Table II. Statistical analysis of particle size analyzer

\begin{tabular}{|c|c|c|c|}
\hline \multicolumn{4}{|c|}{ Volumen estadístico (aritmético) 100\% } \\
\hline & $\mathbf{a}$ & b & c \\
\hline Media & 23,56 & 95,14 & 106,3 \\
\hline Mediana & 17,38 & 78,14 & 94,47 \\
\hline Moda & 23,81 & 127,6 & 127,6 \\
\hline D.S. & 21,10 & 77,26 & 75,35 \\
\hline S.V. & $89,5^{(*)}$ & $81,2^{(*)}$ & $70,9^{(*)}$ \\
\hline$\%<$ & $\mathbf{a}$ & b & c \\
\hline 0 & 2,498 & 10,29 & 17,40 \\
\hline 5 & 7,405 & 31,86 & 46,12 \\
\hline 50 & 17,38 & 78,14 & 94,49 \\
\hline 5 & 33,03 & 140,5 & 151,8 \\
\hline 0 & 56,98 & 207,6 & 212,7 \\
\hline
\end{tabular}

Cálculo de partículas de 0,375 a $948 \mu \mathrm{m}$.

Tamaño en micras, $\left({ }^{*}\right) \%$ 
La figura 2 presenta los resultados obtenidos en la recuperación de oro y plata durante la lixiviación del concentrado, por un periodo de $25 \mathrm{~h}$ sin ningún tratamiento de tostación.

En esta figura se muestra que a las $2,5 \mathrm{~h}$, el oro tiene un porcentaje de recuperación del 24,16\%, posteriormente a las $5 \mathrm{~h}$ se incrementa hasta un $28,89 \%$, alcanzando un máximo del $34,16 \%$ a las $25 \mathrm{~h}$. La plata tiene un comportamiento ligeramente superior, con porcentajes de recuperación de 31,36\% en las primeras 2,5 h, hasta llegar a 40,19\% después de 25 h de lixiviación. El consumo de cianuro para este tipo de concentrado sin tostar alcanza los $3,6 \mathrm{~kg} / \mathrm{m}^{3}$. Se puede apreciar tanto para el oro como para la plata, que las recuperaciones por el método de cianuración de estos concentrados son demasiado bajas, por lo que se considera al concentrado como refractario, siendo necesario utilizar un método complementario y/o alternativo para su tratamiento, con el fin de aumentar la recuperación de los valores de oro y plata.

En la figura 3 se observan los resultados obtenidos en las pérdidas de peso, azufre y arsénico del concentrado calcinado a diferentes temperaturas, de los 350 a $800^{\circ} \mathrm{C}$ con un tiempo de tostación de $1 \mathrm{~h}$.

A temperaturas menores de $350^{\circ} \mathrm{C}$ existe una pérdida de peso no mayor del 0,94\%; al aumentar la temperatura a $400{ }^{\circ} \mathrm{C}$ el incremento en la pérdida de peso aumenta notablemente alcanzando un $13,42 \%$, siguiendo este aumento hasta un 18,68 \% a una temperatura de $450^{\circ} \mathrm{C}$. A partir de esa temperatura, la velocidad de pérdida de peso disminuye, alcanzando un máximo de 19,6 \% a una temperatura de $800^{\circ} \mathrm{C}$. Podemos pensar que a temperaturas alrededor de $450^{\circ} \mathrm{C}$ la pérdida de peso se relaciona con la pérdida de azufre presente en el concentrado, entre otros elementos volátiles.

En el caso del azufre, a temperaturas menores de $350^{\circ} \mathrm{C}$, la eliminación por volatilización del contenido total es bajo, con un $1,14 \%$. Entre 350 y $450^{\circ} \mathrm{C}$ la pérdida de azufre se incrementa rápidamente hasta un $76,3 \%$ y a $500{ }^{\circ} \mathrm{C}$ alcanza un $84,55 \%$. A partir de esta última temperatura, la velocidad de pérdida se hace más lenta hasta alcanzar un $93,16 \%$ a $700{ }^{\circ} \mathrm{C}$. Observamos en la gráfica, que los resultados obtenidos coinciden con los esperados, ya que el punto de ebullición del azufre $\left(444,6^{\circ} \mathrm{C}\right)$, está dentro de los intervalos de temperaturas manejadas.

El porcentaje de pérdida de arsénico a $350^{\circ} \mathrm{C}$ es aproximadamente del $12 \%$, al ir incrementando la temperatura, se aprecia que el porcentaje aumenta de tal manera que a una temperatura de $700{ }^{\circ} \mathrm{C}$ la pérdida es del $40 \%$. Sin embargo, la ley del arsénico en el concentrado es baja, 0,15\%; analizando estos

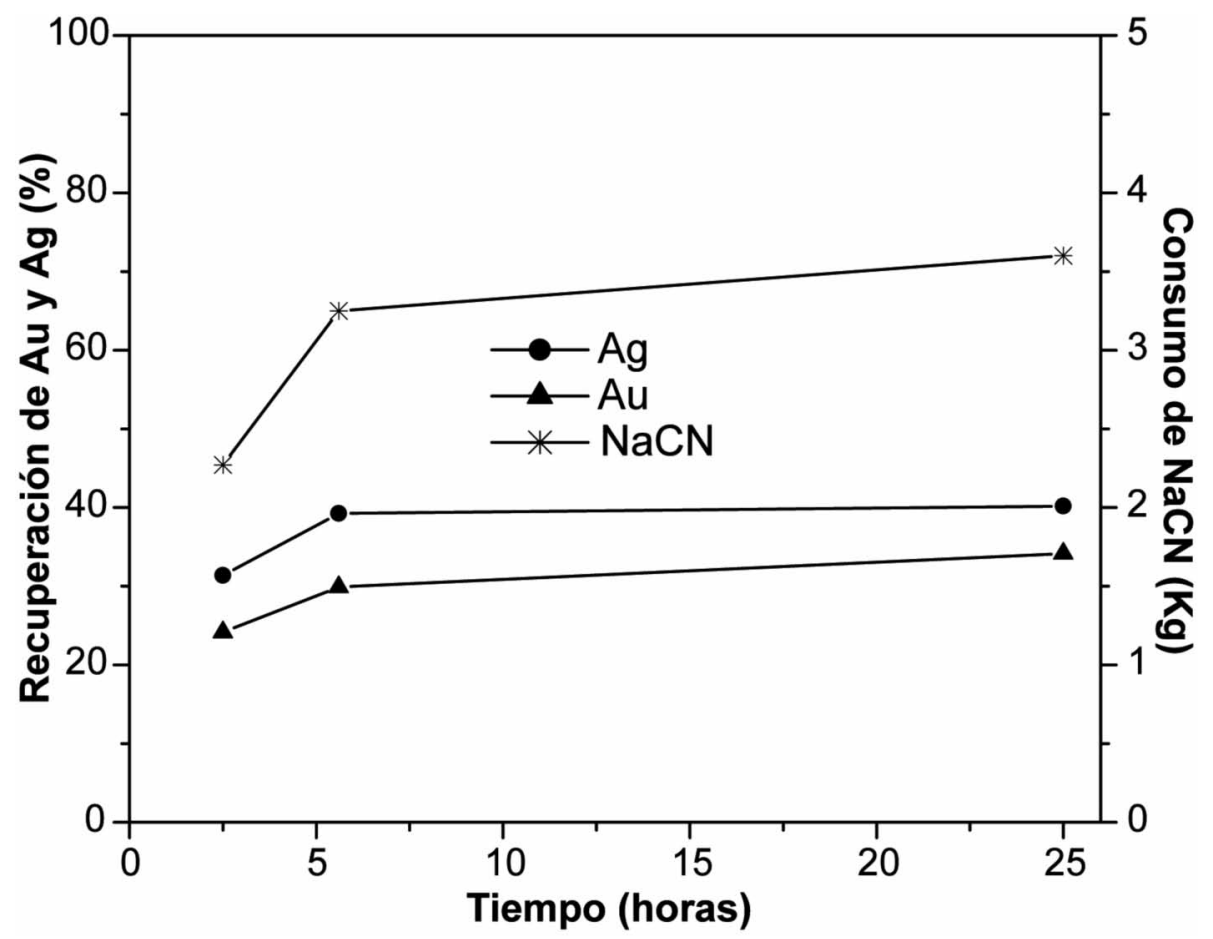

Figura 2. Recuperación de oro y plata y consumo de cianuro del concentrado sin tratamiento de tostación. $\mathrm{t}=25 \mathrm{~h}$, peso de muestra $=12 \mathrm{~g}$.

Figure 2. Recovery of gold and silver, and cyanide consumption from the concentrate without a roasting threatment, $t=25 \mathrm{~h}$, sample weight $=12 \mathrm{~g}$. 


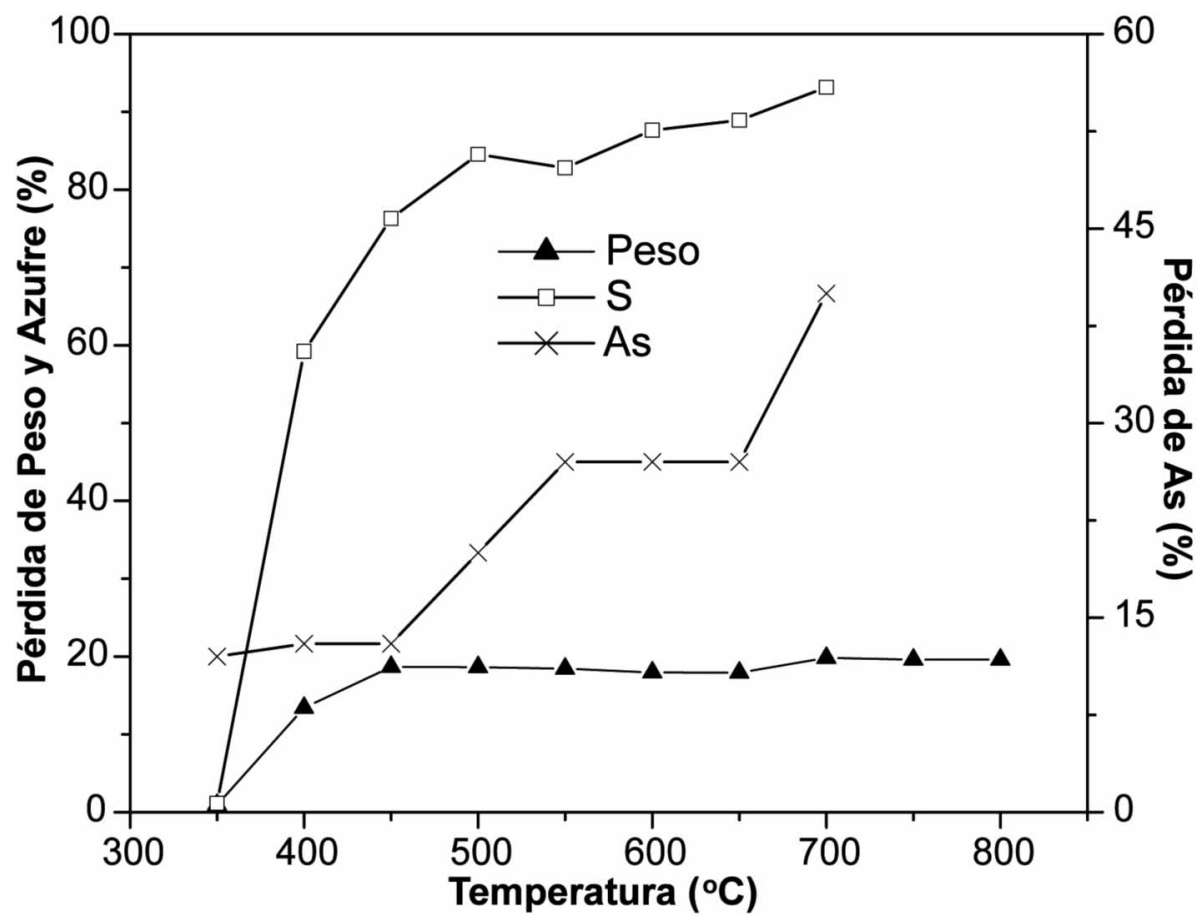

Figura 3. Efecto de la temperatura de tostación, en la pérdida de peso, azufre y arsénico del concentrado, $\mathrm{t}=1 \mathrm{~h}$, peso de muestra $=12 \mathrm{~g}$, $\mathrm{T}=500^{\circ} \mathrm{C}$.

Figure 3. Effect of roasting temperature, in weight loss of sulfur and arsernic from the concentrate, $t=1 \mathrm{~h}$, sample weight $t=12 \mathrm{~g}, T=500^{\circ} \mathrm{C}$.

resultados se puede pensar que el intervalo de temperatura de tostación no afecta de forma general en el contenido de arsénico, por lo que posiblemente éste se encuentra asociado con alguna especie que no permite su completa eliminación.

En la figura 4 se muestran los resultados del comportamiento en la recuperación de oro y plata de concentrado tostado y lixiviado durante $1 \mathrm{~h}$. La recuperación del oro a temperaturas de tostación de $450^{\circ} \mathrm{C}$ es del $32,16 \%$; al aumentar la temperatura a $500^{\circ} \mathrm{C}$ existe una disminución drástica en los porcentajes de recuperación del oro de 10,83\%, hasta llegar a $6,58 \%$ a $700{ }^{\circ} \mathrm{C}$. Los porcentajes de lixiviación de la plata se encuentran alrededor del $10,68 \%$ a 450 ${ }^{\circ} \mathrm{C}$, manteniendo valores casi similares hasta la temperatura de $700^{\circ} \mathrm{C}$. El consumo de cianuro de sodio es de alrededor de los $0,5 \mathrm{~kg} / \mathrm{m}^{3}$ a $400{ }^{\circ} \mathrm{C}$, generándose un aumento paulatino hasta llegar alrededor de $\operatorname{los} 0,8 \mathrm{~kg} / \mathrm{m}^{3}$ a $700^{\circ} \mathrm{C}$.

Puede observarse, que tanto las recuperaciones de plata como las de oro son muy bajas; posiblemente los tiempos de tostación y lixiviación no son los adecuados. La calcina que se obtiene en la tostación es de color gris-negruzco, indicándonos que hay presencia de una mayor cantidad de magnetita, lo cual no favorece a la lixiviación, coincidiendo con lo observado en otros estudios, donde un color de calcina café-chocolate indica una mayor presencia de hematita $(\approx 75-80 \%)$, siendo el resto de magnetita, donde se obtienen las mejores recuperaciones ${ }^{[9]}$.

La figura 5 presenta los resultados obtenidos en la recuperación de oro y plata del concentrado tostado y lixiviado con un tiempo de $4 \mathrm{~h}$ de tostación y $20 \mathrm{~h}$ de lixiviación respectivamente.

En esta figura puede observase que el oro se recupera aproximadamente en un $27 \%$ a una temperatura de tostación entre 400 y $500^{\circ} \mathrm{C}$. Al aumentar la temperatura a $600^{\circ} \mathrm{C}$ hay un aumento en la recuperación de oro casi del $50 \%$, y al tostar a $700{ }^{\circ} \mathrm{C}$ la recuperación disminuye drásticamente hasta un 20,66 \%. Respecto a la plata, ésta sigue una tendencia parecida al oro, pero con una mayor recuperación, así podemos observar, que al tostar el concentrado a $400^{\circ} \mathrm{C}$, la recuperación es del $31,32 \%$, alcanzando un máximo de $61,27 \%$ a $600{ }^{\circ} \mathrm{C}$. Al incrementar la temperatura a $700^{\circ} \mathrm{C}$ existe una caída en la recuperación de plata al $51 \%$. La pérdida de la recuperación del oro y plata a altas temperaturas, posiblemente se deba a la sinterización de la partícula de concentrado.

El consumo de cianuro a $400^{\circ} \mathrm{C}$, es de $0,8 \mathrm{~kg} / \mathrm{ton}$ y disminuye hasta un $0,2 \mathrm{~kg} / \mathrm{m}^{3}$ a $700^{\circ} \mathrm{C}$.

En la figura 6 se presentan los resultados obtenidos en la recuperación de oro y plata, cuando se agregó inmediatamente el concentrado tostado (calcina), 


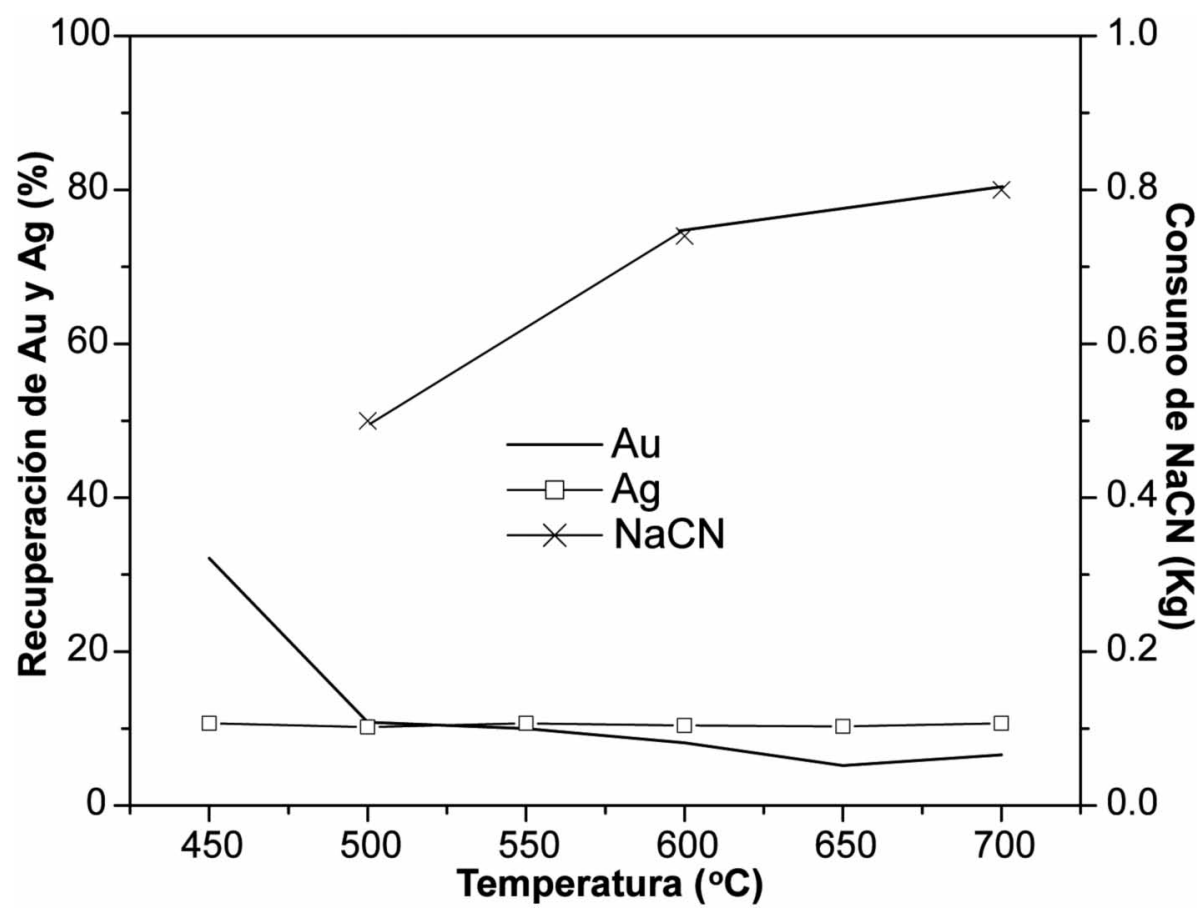

Figura 4. Resultados en la recuperación de oro, plata y consumo de cianuro del concentrado tostado y lixiviado, $\mathrm{t}=1 \mathrm{~h}$, peso de muestra $=12 \mathrm{~g}$.

Figure 4. Recovery of gold and silver, and cyanide consumption from the concentrate roasted and leached, $t=1 \mathrm{~h}$, sample weight $=12 \mathrm{~g}$.

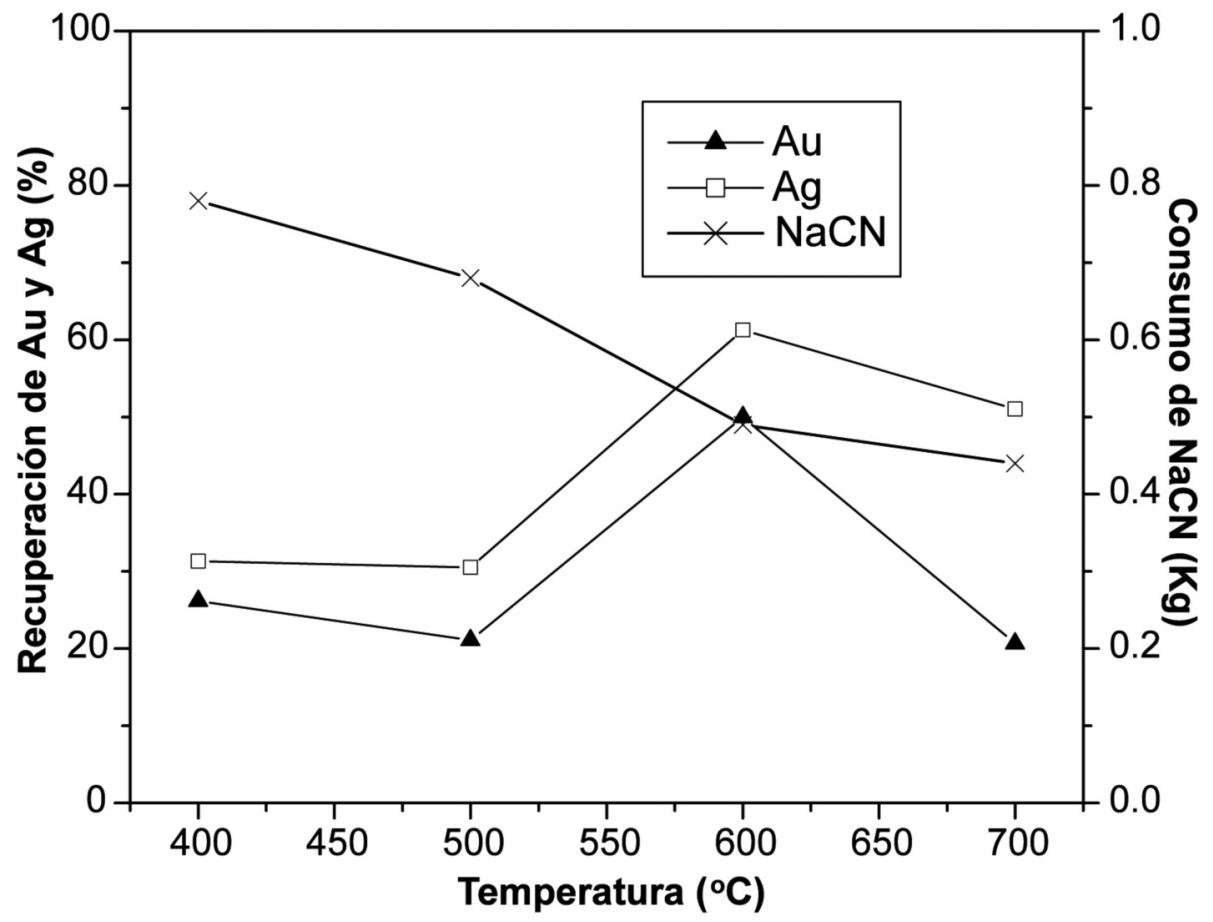

Figura 5. Resultados en la recuperación de oro, plata y consumo de cianuro del concentrado tostado y lixiviado, tostación $t=4 \mathrm{~h}$, lixiviación $\mathrm{t}=20 \mathrm{~h}$, peso de muestra $=12 \mathrm{~g}$.

Figure 5. Recovery of gold and silver, and cyanide consumption from the concentrate roasted and leached roasting treatment, $t=4 \mathrm{~h}$, leaching $t=20 \mathrm{~h}$, sample weight $=12 \mathrm{~g}$. 


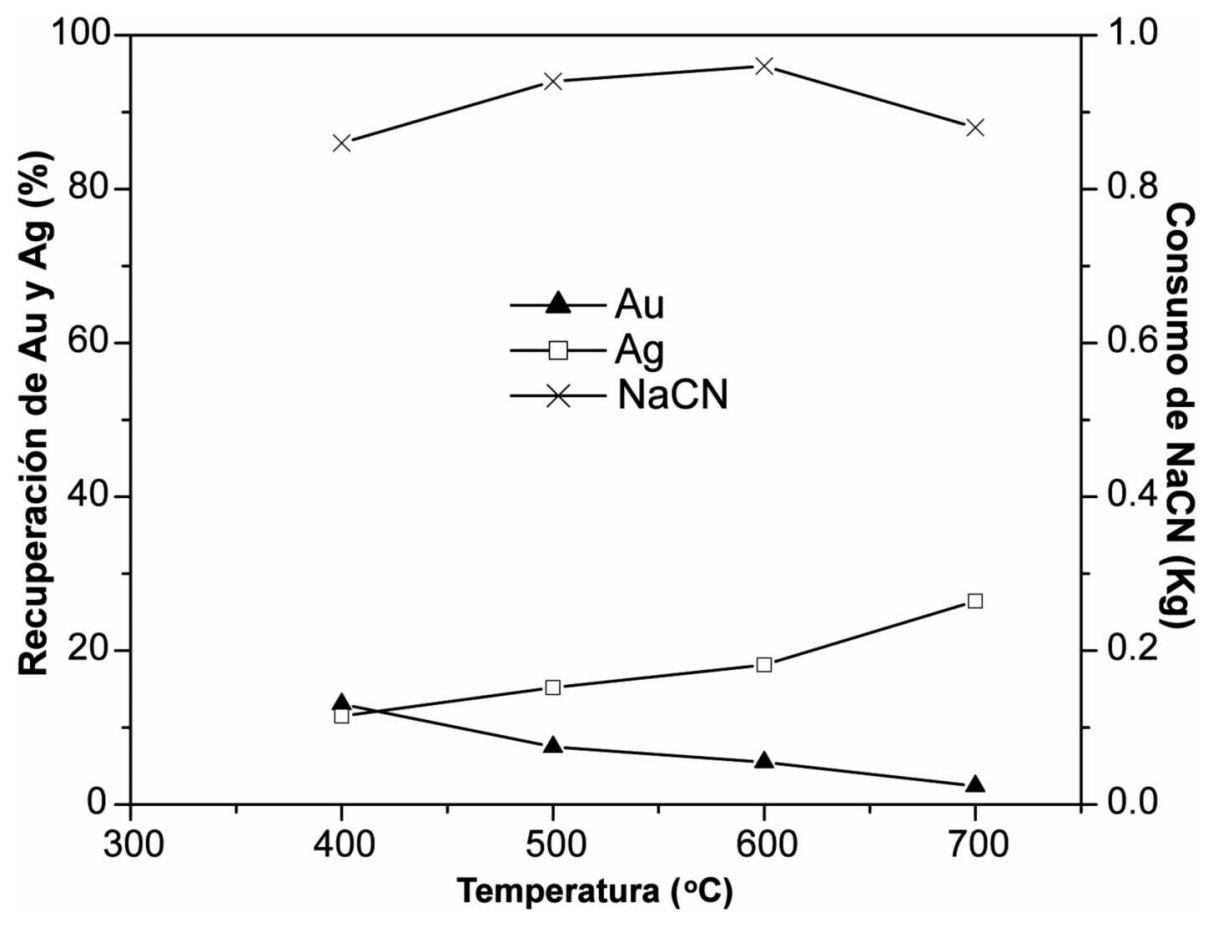

Figura 6. Resultados en la recuperación de oro, plata y consumo de cianuro del concentrado tostado y lixiviado, tostación $t=4 \mathrm{~h}$, lixiviación a una temperatura inicial de $40^{\circ} \mathrm{C}, \mathrm{t}=20 \mathrm{~h}$, peso de muestra $=12 \mathrm{~g}$.

Figure 6. Recovery of gold and silver, and cyanide consumption of the concentrate roasted and leached roasting treatment, $t=4 \mathrm{~h}$, leaching at initial temperature of $40^{\circ} \mathrm{C}, t=20 \mathrm{~h}$, sample weight $=12 \mathrm{~g}$.

desde el horno a la solución lixiviante, que se encontraba a $40^{\circ} \mathrm{C}$, con el objetivo de observar el comportamiento en los porcentajes de recuperación del oro y plata al efectuar un cambio brusco en la temperatura de la calcina caliente. La recuperación del oro a la temperatura de $400^{\circ} \mathrm{C}$ se encuentra alrededor del $13 \%$; al aumentar la temperatura disminuye la extracción de tal manera que a $500^{\circ} \mathrm{C}$ es aproximadamente $7 \%$, hasta llegar a un $3 \%$ en $700{ }^{\circ} \mathrm{C}$. Por otro lado, la recuperación de plata se encuentra en $11 \%$ a $400{ }^{\circ} \mathrm{C}$, empezando a subir a $15 \%$ en $500^{\circ} \mathrm{C}$, para alcanzar un $26 \%$ a $700{ }^{\circ} \mathrm{C}$.

Estas recuperaciones de oro y plata son demasiado bajas, ya que la recuperación del oro cae hasta un $3 \%$, y aunque la plata sube, en general es muy poco. Se observa que a diferencia de las pruebas anteriores el comportamiento es muy diferente, posiblemente el cambio de temperatura de forma rápida afecta a la superficie de la partícula, lo cual impide la penetración de la solución lixiviante para la extracción de los valores. Existe un aumento de la temperatura en la solución de 2 a $3{ }^{\circ} \mathrm{C}$, lo cual no provoca beneficio alguno para la etapa de lixiviación en este caso. El consumo de cianuro oscila en un promedio de $0,9 \mathrm{~kg} / \mathrm{m}^{3}$.
En la figura 7 se presentan los resultados de la recuperación de oro y plata cuando la calcina caliente se pone en contacto con la solución lixiviante que se encuentra a una temperatura de $50^{\circ} \mathrm{C}$. Los valores obtenidos son casi similares a los obtenidos en la gráfica anterior (Fig. 6), ya que el porcentaje de recuperación de oro se encuentra alrededor del $24 \%$ a una temperatura de tostación de $400^{\circ} \mathrm{C}$, y disminuye hasta un $1,5 \%$ a $700{ }^{\circ} \mathrm{C}$. La recuperación de plata a $400{ }^{\circ} \mathrm{C}$ es del $10 \%$ y sufre un incremento hasta un $32 \%$ a $700{ }^{\circ} \mathrm{C}$. Con lo anterior se constata que al agregar la calcina caliente directamente del horno a la solución lixiviante, no existe una mejora en la recuperación del oro, sino que la perjudica notablemente, ya que las recuperaciones son demasiado bajas. Por otro lado, aunque la recuperación de plata se incrementa, estos resultados no son del todo satisfactorios. El consumo de cianuro se encuentra alrededor de $0,8 \mathrm{~kg} / \mathrm{m}^{3}$.

En la figura 8 se presenta el consumo de cal para elevar el $\mathrm{pH}$ del concentrado lixiviado en $24 \mathrm{~h}$, alrededor de $1,3 \mathrm{~kg} / \mathrm{m}^{3}$ para subir el $\mathrm{pH}$ a 11,4. También se presenta el consumo de cal de la lixiviación del concentrado tostado a $500^{\circ} \mathrm{C}$. $\mathrm{Al}$ inicio el pH de la solución oscila alrededor de 3,0; al agregar el equi- 


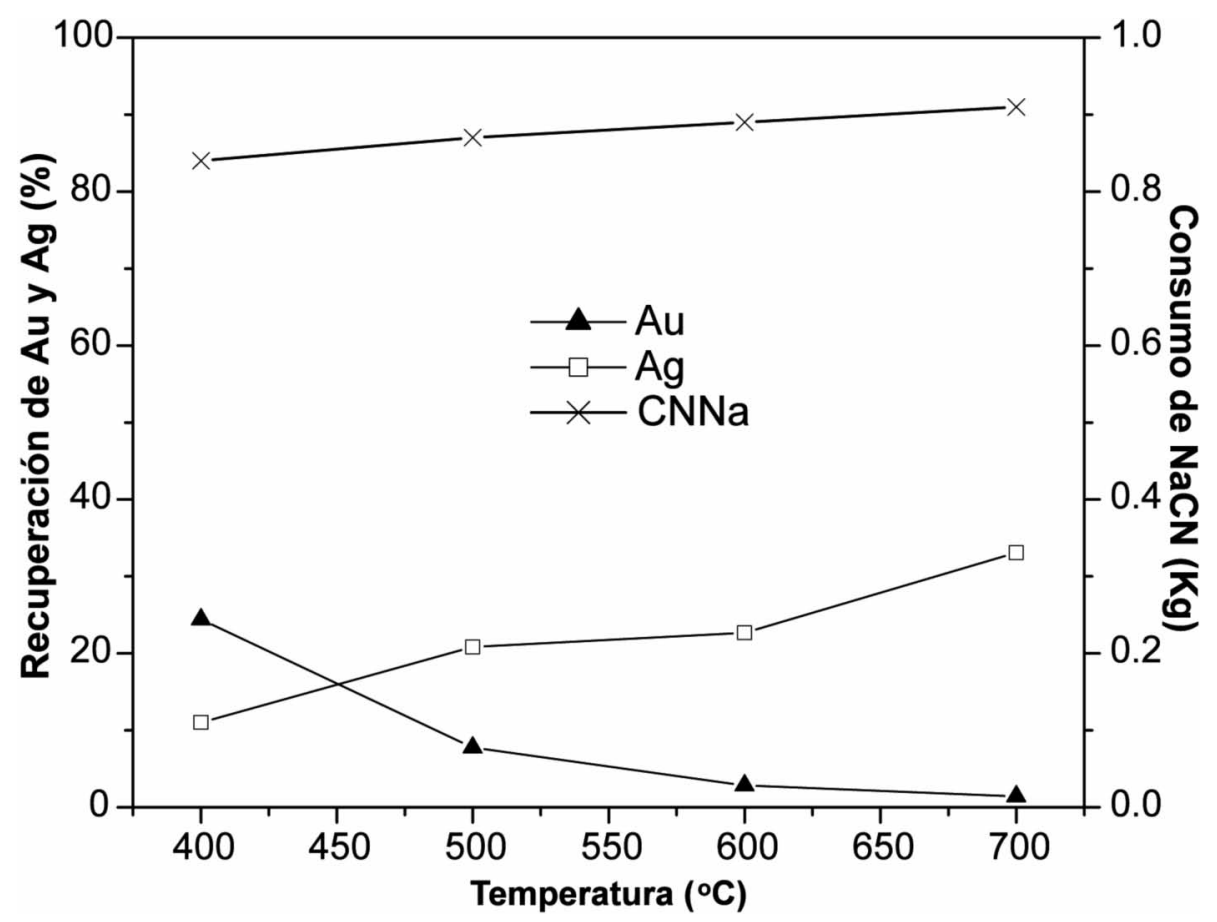

Figura 7. Resultados en la recuperación de oro, plata y consumo de cianuro del concentrado tostado y lixiviado, tostación $t=4 \mathrm{~h}$, lixiviación a una temperatura inicial de $50^{\circ} \mathrm{C}, \mathrm{t}=20 \mathrm{~h}$, peso de muestra $=12 \mathrm{~g}$.

Figure 7. Recovery of gold and silver, and cyanide consumption of the concentrate roasting and leached roasting $t=4 \mathrm{~h}$, leaching at initial temperature of $50^{\circ} \mathrm{C}, t=20 \mathrm{~h}$, sample weight $=12 \mathrm{~g}$.

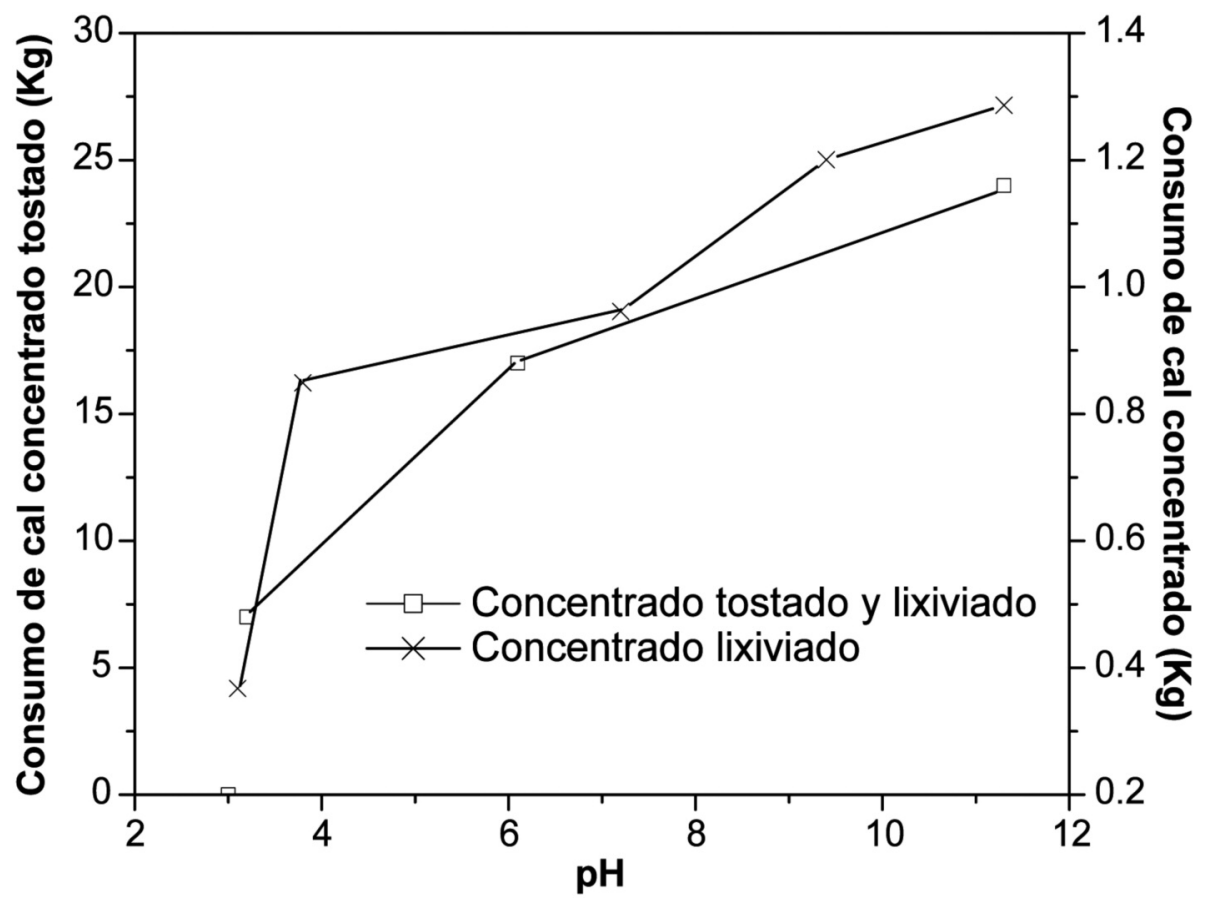

Figura 8. Consumo de cal del concentrado lixiviado y tostado. Lixiviación $\mathrm{t}=20 \mathrm{~h}$, tostación $\mathrm{t}=4 \mathrm{~h}$, peso de muestra $=12 \mathrm{~g}$.

Figure 8. Consumption of limestone from the concentrate leached and roasted. Leaching $t=20 \mathrm{~h}$, roasting $t=4 \mathrm{~h}$, sample weight $=12 \mathrm{~g}$. 
valente a $7 \mathrm{~kg} / \mathrm{m}^{3}$ de cal el $\mathrm{pH}$ es de 3,2; al aumentar la cal a $17 \mathrm{~kg} / \mathrm{m}^{3}$ el $\mathrm{pH}$ es de 6,1 , siendo necesario agregar otros $7 \mathrm{~kg}$ de cal (suman ya $23 \mathrm{~kg} / \mathrm{m}^{3}$ ) para llegar a un $\mathrm{pH}$ mayor de 11 . Si comparamos el consumo de cal para elevar el $\mathrm{pH}$ alrededor de 10,5 con respecto a un mineral oxidado, éste requiere aproximadamente $1 \mathrm{~kg} / \mathrm{m}^{3}$, por lo que concluimos que es demasiada la cal necesaria para obtener el $\mathrm{pH}$ adecuado para la lixiviación.

\section{CONCLUSIONES}

- El concentrado presenta una ley de oro de $60 \mathrm{~g} / \mathrm{t}$ y de plata de $10,200 \mathrm{~kg} / \mathrm{t}$, un $36,38 \%$ de hierro y $33,34 \%$ de azufre. El azufre se elimina hasta un $60 \%$ a una temperatura de tostación inicial de $400{ }^{\circ} \mathrm{C}$, hasta alcanzar un $93 \%$ a una temperatura de $700{ }^{\circ} \mathrm{C}$.

- Al tostar el concentrado se detectó un incremento en el tamaño de partícula, debido posiblemente a la eliminación del azufre en forma de gas. La lixiviación del concentrado por 24 h, alcanza un porcentaje de recuperación del oro del $34 \%$ y del $40 \%$ para la plata, confirmándose la clasificación de mineral como refractario.

- A una temperatura de tostación de $600^{\circ} \mathrm{C}$ y después de lixiviar con cianuro de sodio se obtiene un porcentaje de recuperación máxima del oro del $50 \%$ y $61 \%$ para la plata. Al aumentar la temperatura a $700{ }^{\circ} \mathrm{C}$, las recuperaciones disminuyen, posiblemente debido a una sinterización de la partícula. Al someter la calcina caliente, a la solución lixiviante, existe un aumento de la solución de 2 a $3{ }^{\circ} \mathrm{C}$, resultando en porcentajes de recuperación de oro y plata muy bajos.

- El consumo de cal para elevar el $\mathrm{pH}$ a las condiciones de lixiviación con cianuro $(\mathrm{pH}>10)$, oscila alrededor de $25 \mathrm{~kg} / \mathrm{m}^{3}$. El color de la calcina café-chocolate, nos indica que se efectúa una oxidación de los sulfuros a hematita $(75-80 \%)$ y magnetita (20-25\%) lo cual favorece a la lixiviación, coincidiendo con lo observado en otros estudios $^{[9]}$, donde reportan buenas recuperaciones del mineral de oro y plata. Se recomienda hacer otras pruebas con otros lixiviantes, para comparar recuperaciones, y/o utilizar otro método alternativo en lugar de tostación.

\section{REFERENCIAS}

[1] E. Salinas, I. Rivera, F. R. Carrillo, F. Patiño, J. Hernández y L. E. Hernández. Rev. Soc. Quím. Méx. 48 (2004) 315-320.

[2] J. R. Parga y F. R. Carrillo. Rev. Metal. Madrid, 32 (1996) 254-261.

[3] V. Arias-Arce, R. Coronado-Falcón, L. Puente Santibáñez y D. Lovera-Dávila. Revista del Instituto de Investigación FIGMMG, 8 (2005) 5-16.

[4] O. Celep, I. Alp, H. Deveci y M. Vicil. Met. China 19 (2009) 707-713.

[5] J. H. Coronado, Tesis de Maestría, Departamento de Ingeniería Química y Metalurgia, Universidad de Sonora, 1997.

[6] J. R. Goode. TMS, EPD Congress, 1993, pp. 291-317.

[7] J. C. Yannopoulus. The extractive metallurgy of gold, Van Nostrand Reinhold, 1991 pp. 79-98.

[8] D. C. Harris. Mineral. Deposita 25 (1990) 3-7.

[9] R. B. Coleman. Proceedings of Gold. Symposum, 1990, pp. 381-388.

[10] A. Vladimir, R. C. Falcon, L. Puente y D. Lovera. Rev. Inst. Inv. FIGMMG, 16 (2005) 5-7.

[11] P. R. Taylor, Z. B. Yin y R. W. Barlett. Gold 90, SME, 1990, pp. 411-419. 